\title{
Situation de la parole dans l'enseignement moral et civique
}

Situation of Speech in Moral and Civic Education

\section{Myriam Belmonte-Estrade}

\section{OpenEdition}

Journals

Édition électronique

URL : http://journals.openedition.org/recherchestravaux/1584

DOI : $10.4000 /$ recherchestravaux. 1584

ISSN : 1969-6434

Éditeur

UGA Éditions/Université Grenoble Alpes

Édition imprimée

ISBN : 978-2-37747-098-3

ISSN : 0151-1874

Référence électronique

Myriam Belmonte-Estrade, "Situation de la parole dans l'enseignement moral et civique », Recherches \& Travaux [En ligne], 94 | 2019, mis en ligne le 20 juin 2019, consulté le 08 septembre 2020. URL:

http://journals.openedition.org/recherchestravaux/1584; DOI : https://doi.org/10.4000/

recherchestravaux.1584

Ce document a été généré automatiquement le 8 septembre 2020

(c) Recherches \& Travaux 


\title{
Situation de la parole dans l'enseignement moral et civique
}

\author{
Situation of Speech in Moral and Civic Education \\ Myriam Belmonte-Estrade
}

1 Alors que nous n'avons jamais autant communiqué, jamais la parole enseignante n'a été aussi rare, en particulier la parole qui transmettrait un enseignement moral ou civique. Par parole, nous entendons l'usage singulier et individualisé de la faculté de parler où une personne s'adresse à une autre personne. En devenant parole, le discours s'incarne et porte à la fois un sens et une promesse car celui qui le prononce y est engagé. Or, en dépit des nombreuses incantations et exhortations, grand est de nos jours le silence autour des valeurs ou des vertus morales et civiques. S'il y a une expression péjorative dans le français contemporain, c'est bien l'expression «faire la morale » et celui qui se risquerait à cette tentative se mettrait rapidement dans une position ridicule, voire suspecte, et, de plus, vaine, tant et si bien qu'au mot discrédité de "morale » on lui préfère bien souvent celui d'«éthique »: la morale ou comment en parler sans en parler. Cette disqualification atteint son apogée quand on prétend l'enseigner. Comment dès lors "parler de morale sans faire de morale ${ }^{1}$ »? Afin d'analyser cette question nous tenterons d'abord de décrire la situation de la parole, d'en proposer ensuite quelques éléments d'analyse, pour envisager enfin dans quelle mesure l'étude de la littérature, en offrant un espace de transmission et de transfert, peut contribuer à l'éducation morale et civique.

\section{D'un risque de « délaissement »}

2 Si on tente de décrire la situation contemporaine de la parole dans l'enseignement moral et civique, c'est d'abord de silence dont il faudrait parler tant on est immédiatement frappé par la difficulté à parler de morale. Quand il s'agit d'affirmer des valeurs et de les faire partager, beaucoup d'adultes semblent éprouver une grande gêne. Mal assurés de leurs propres positions, fragilisés dans un monde de plus en plus instable, ils peinent à les transmettre. Chute des idéologies, recul du religieux, 
ouverture multiculturelle contribuent à un effacement des cadres normatifs et imposent un relativisme généralisé. Toutes les positions semblent frappées d'arbitraire, tant et si bien qu'au nom de la tolérance et de "l'ouverture aux autres", l'universalisme semble présomptueux. Il ne s'agit pas là seulement d'une prudence un peu molle ou du conformisme du " politiquement correct », mais plutôt d'un réel souci éthique qui conduit chacun à une modestie et à une retenue bienveillante. On objectera sans doute que certains se font entendre bruyamment. Or, ces manifestations, dont certaines prétendent "libérer la parole ", la musellent plutôt, tant l'opinion publique, dans son ensemble, rejette, et c'est heureux, le dogmatisme. Or, ce qui est sans doute un progrès devient problématique lorsqu'il s'agit d'éduquer. Comment un parent ou un éducateur pourrait-il exprimer des convictions s'il n'en est pas lui-même convaincu? Comment pourrait-il s'autoriser à contraindre, et même à interdire, au nom de principes, d'idéaux et de finalités éducatives, s'il a perdu tout horizon de sens et ne peut dès lors même plus s'imposer d'obligations à lui-même ? La transmission a tôt fait d'être confondue avec une insupportable imposition, ce qui conduit en fait à un « refus de l'éthique ${ }^{2}$ ", par honnêteté intellectuelle et morale.

3 À l'école, la prise de parole des enseignants est encore plus délicate. Les devoirs du fonctionnaire, la laïcité, la posture professionnelle calment les velléités. Soumis à des injonctions multiples et parfois contradictoires (favoriser la co-éducation, mais émanciper les élèves de leurs familles, par exemple), les enseignants pêchent souvent par excès de zèle. Ainsi la neutralité laïque et le droit de réserve tendent à être interprétés de manière maximaliste ce qui aboutit à cette "laïcité silence ", "laïcité abstention $^{3}$ » ou " d'ignorance ${ }^{4}$ » où, pour ne pas heurter les croyances et malmener les consciences, on n'ose plus aborder aucun sujet moral, politique ou existentiel : l'enfer est pavé de bonnes intentions... La voie est donc bien étroite entre le trop et le pas assez et la manière difficile à trouver. Tantôt on rassure l'instituteur en lui disant qu'on ne lui demande pas de se transformer en professeur de morale, en "apôtre d'un nouvel évangile", en "théologien" ou en "philosophe improvisés"), tantôt on semble disqualifier par avance toute tentative en lui assénant qu'il "ne pourra jamais remplacer le curé ou le pasteur, même s'il est important qu'il s'en rapproche ${ }^{6} »$. Il y a de quoi être découragé. En dépit des réformes et des incitations louables, la morale risque dès lors toujours d'être le parent pauvre de l'EMC. Instruire des institutions républicaines passe encore, mais enseigner la morale, c'est autre chose. Parce que le lien consubstantiel entre morale, vertus civiques et idéaux démocratiques s'est relâché, parce que l'écart entre les principes et la réalité est bien grand, la morale à l'école risque d'être perçue encore, et pour longtemps, comme un supplément d'âme.

4 Cependant, cette frilosité des adultes peut être cher payée. Privés d'interdits et de repères, les enfants ne sauraient grandir et s'élever en accédant au symbolique, puisque c'est par la parole qui inter-dit, qui interpose un dire entre la pulsion et sa réalisation, qu'ils s'humanisent; il est nécessaire pour cela d'instituer un rapport nourricier à la culture et au passé (ce qui ne veut pas dire qu'on soit conservateur, rétrograde ou réactionnaire). Le silence des adultes peut devenir coupable ; il n'est plus alors le signe de leur délicatesse ou leur prudence, mais de leur lâcheté et de leur démission. Ce refus de la transmission, cette forme bien particulière d'abandon des enfants, en dépit de tous les soins qu'on leur prodigue par ailleurs, événement dans l'histoire de l'humanité, appelait une dénomination nouvelle : H. Arendt le désigne comme « délaissement ». Il est donc urgent de réfléchir à la crise de la transmission et à notre rapport aux 
générations futures, à l'absence de perspectives porteuses d'horizon, d'initiations qui caractérise trop souvent les relations entre adultes et enfants et à ce que signifie cette défiance et cette dévaluation du rapport humain, ce qu' $\mathrm{H}$. Arendt formule en ces termes : «L'autorité a été abolie par les adultes et cela ne peut que signifier une chose : que les adultes refusent d'assumer la responsabilité du monde dans lequel ils ont placé les enfants ${ }^{7}$."

Or, cette question n'est pas seulement pédagogique et, en ce sens, elle ne concerne pas seulement les spécialistes en sciences de l'éducation. Il y va de la préservation d'une vie démocratique ou même, tout simplement, politique, d'une vie en commun et c'est pourquoi l'éducation morale suppose aussi que soient préservées l'autorité des adultes, la possibilité d'une transmission, la circulation de la parole entre les générations, bref tout ce qui lie les hommes ici et maintenant à une histoire et à un avenir. Pour cela, il faut que les attaches au passé n'aient pas été rompues et qu'ils partagent un même espoir, ce que justement $\mathrm{H}$. Arendt appelle un «monde commun » et qui serait selon elle particulièrement menacé.

\section{Une parole « horizontale » ne suffit pas}

6 Or on assiste aujourd'hui à une extraordinaire promotion d'une parole «horizontale ", c'est-à-dire entre pairs, notamment entre enfants, au détriment d'une parole "verticale ", de l'adulte à l'enfant, du représentant de l'autorité à celui qui y est subordonné. Cette évolution témoigne des aspirations profondes d'une société moderne, démocratique et individualiste, mais révèle aussi nos difficultés à parler de la morale et $a$ fortiori à l'enseigner. Ainsi le programme de l'EMC ${ }^{8}$ mis en œuvre depuis la rentrée 2015 encourage vivement l'usage des débats entre élèves, où l'enseignant a essentiellement un rôle de modérateur, et ce, dès l'école primaire. Certes, les discussions à visée philosophique (DVP) constituent une pratique pédagogique très stimulante et tout à fait pertinente en EMC ; les élèves font souvent preuve d'une acuité de jugement qui nous étonne et de capacités réflexives que nous ne leur accordions pas toujours, mais l'enthousiasme qu'ils suscitent ne doit pas nous empêcher d'affronter les problèmes de fond qu'ils soulèvent, ni de nous interroger sur ce qu'ils pourraient déceler'. En effet, si on fait vraiment de la philosophie, cette pratique ne manque pas d'être déstabilisante et on peut légitimement se demander si nous avons le droit d'infliger cela à des enfants et de les placer, par exemple, dans un conflit de loyauté par rapport à leurs parents, car il est bien difficile d'apprécier la part de spontanéité dans la parole des élèves. Dans bien des cas, cela met les enseignants dans une position délicate où ils s'interrogent sur la posture à adopter. Mais, plus essentiellement, n'eston pas d'autant plus enclins à s'emparer de ces discussions, qu'elles apparaissent comme une des rares ressources disponibles, qui nous permettraient d'éviter ces écueils que nous redoutons tant, le dogmatisme et la magistralité, mais peut-être aussi, de façon moins avouable, parce qu'en nous en remettant à nos élèves, nous serions déchargés d'une tâche que nous avons bien du mal à accomplir, d'abord pour nousmêmes, ce souci de donner un sens à nos existences, de porter un jugement, de tenir une parole qui nous engage? Mais est-ce bien aux enfants de le faire? Quand sous nos yeux éblouis ils débattent en cercle en se faisant passer un bâton de parole, ce que nous admirons parfois n'est-ce pas aussi cette créativité philosophique, cette capacité à 
inventer des valeurs, à rêver la vie, toutes ces dispositions que nous avons perdues, nous qui n'osons plus penser?

7 A minima, on peut relever ici une certaine ambivalence de la part des adultes, qui va de pair avec une valorisation très ambiguë de la figure de l'enfant. En effet, la reconnaissance de la spécificité de l'enfant accroit tellement la distance avec l'adulte qu'elle le rend inaccessible, ce qui conduit soit à l'infantiliser et à le maintenir indéfiniment dans l'enfance, l'incursion des adultes dans le monde des enfants ne pouvant être vécue que comme une infraction susceptible de créer des blessures irrémédiables ; soit, au contraire, à méconnaître ses besoins éducatifs et à le considérer comme d'emblée autonome, comme si l'autonomie n'avait pas besoin d'être instituée (comme en témoigne les débats récents autour de la justice des mineurs). Cependant, à ces enfants "en mal d'enfance ${ }^{10}$ » correspondent peut-être des adultes puérils et éternels mineurs. Les âges de la vie sont sans doute devenus très labiles et sont de moins en moins marqués par des rituels de passage, mais on ne peut s'empêcher de penser qu'une société de consommation tributaire pour fonctionner de nos pulsions d'achat, qui capte de plus en plus difficilement notre attention et qui doit autant que possible fidéliser des clients du berceau à la tombe doit permettre aux enfants de prendre le pouvoir et, pour ce faire, doit destituer les adultes de leur autorité éducative. Comme le montre Bernard Stiegler, on y parvient "en découpant les générations en de telles "tranches" qui sont aussi des cibles ${ }^{11}$ " et en humiliant sciemment les adultes dans les représentations qui en sont faites (comme par exemple dans une publicité pour Canal J que Bernard Stiegler analyse). On comprend pourquoi, dans ces conditions, l'éducation morale est désormais devenue si difficile.

8 Pourtant, si on peut plébisciter une circulation plus fluide d'une parole plus horizontale et des rapports entre générations moins crispés, nous ne pouvons pas nous passer entièrement de la tradition et de l'autorité, et donc d'une parole verticale : il faut bien que les plus jeunes puissent continuer à faire confiance aux adultes et trouver dans le monde une place où leur existence puisse prendre un sens, faute de quoi ils risquent de se tourner vers ceux qui leur en proposent une et qui ne sont pas retenus par nos scrupules. Les individus, parce qu'ils trouvent peu de ressources dans la communauté et dans la transmission, doivent tout construire par eux-mêmes : il appartient à chacun de se forger une personnalité, de choisir ses valeurs, donner un sens à sa vie. Tel est leur lot dans nos sociétés démocratiques et "désenchantées». Bref, l'existence personnelle y devient une aventure formidable, mais aussi beaucoup plus solitaire et angoissante. Cependant, le pas à franchir entre l'autonomie postulée et l'autonomie réelle est immense. En fait, plus une société proclame cette autonomie, plus la construction subjective et sociale de l'individualité devient difficile et fragile, puisqu'on a détruit dans le même temps les structures susceptibles de l'étayer, ce que Marcel Gauchet résume par la formule suivante :

Le grand paradoxe de l'anthropologie des modernes, c'est l'ébranlement de l'intime possession de soi-même qui accompagne la conquête de la maîtrise politique individuelle. Plus l'homme est libre extérieurement, plus il est intérieurement

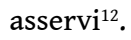

Néanmoins, jusqu'à une époque relativement récente, l'individu pouvait s'inscrire dans un projet collectif de progrès et de libération où l'on postulait que l'homme ne pouvait s'accomplir que dans un parcours vers l'autonomie morale et civique et dans la vie politique : seul le citoyen était, de ce point de vue, un être humain accompli. Certes, ce projet avait son envers: l'individu était tenu de s'émanciper; la conquête de 
l'autonomie était un devoir. Dès lors, le citoyen se constituait en s'arrachant aux déterminations antérieures et privées (familiales, sociales) et l'éducation était pensée comme une rupture par rapport aux déterminations naturelles ou sociales. Aujourd'hui, une telle perspective historique a disparu et plus rien ne vient supporter cette construction. Elle n'apparaît même plus comme désirable. L'individualisme n'est plus pensé comme "affirmatif» et "émancipateur", il perd son côté subversif et apparaît comme « davantage subi que voulu ${ }^{13}$ ». C'est ainsi que l'on peut comprendre la fascination exercée par des fondamentalistes, voire par les fanatismes des plus violents (même si cette seconde tendance ne doit pas être confondue avec la première). Ces manifestations en sont d'autant plus visibles que les individus ont recours à ce qu'il y a de plus structurant pour eux et de plus démonstratif pour les autres. Ils procèdent en piochant des éléments parmi les biens symboliques disponibles, puisqu'il s'agit de se repérer soi-même et d'être repéré par les autres, par le marquage du corps par exemple (c'est ce que le voile nous voile). La croyance devient une identité : elle permet de distinguer un "nous » des " autres ». Par conséquent, il ne faut pas s'étonner que des jeunes gens se tournent vers les formes de paroles les plus verticales, ni vers les formes de religiosité les plus dogmatiques et autoritaires, celles qui rejettent toute interprétation et toute modernité, ni encore vers les communautés les plus fusionnelles et englobantes. En ce sens, ces stratégies d'appropriation du religieux ne constituent en rien un retour à la religion. Il est donc urgent que les adultes et l'école s'emparent des questions morales et existentielles et n'abandonnent pas la transmission, face à ce que Guy Coq appelle des « sacralités barbares de remplacement » qu'il décrit ainsi :

À négliger cette part [éthique] des communautés historiques dans notre réflexion, je crains que nous laissions inoccupée une fonction ancienne du sacré, créant un vide menaçant par la place offerte au retour des sacralités barbares de remplacement.

[...] Si l'instance culturelle n'est pas assurée, des systèmes de substitution relaient la vie culturelle brisée, la conscience commune invisible, par des unifications infrahumaines, voire inhumaines, dans le fantasme de la race, dans l'obsession de la pureté raciale. Si la société ne sait pas dire, ne sait plus communiquer et faire aimer ce qui la tient vivante, reviennent donc les remplacements inhumains d'un lien social défait ${ }^{14}$.

Nous constatons donc une situation caractérisée par une parole rare, plutôt horizontale, et, dans ce silence relatif, émergent de façon menaçante les paroles verticalisées à l'extrême des « sacralités barbares de remplacement ».

\section{Quelques facteurs sociaux du retrait de la parole enseignante}

11 Trois phénomènes semblent concourir à cette situation: la crise de l'autorité, l'émergence d'une société gestionnaire et une méfiance généralisée envers la transmission. Bien entendu, il ne s'agit ni de les déplorer ni de s'en réjouir, mais seulement de les comprendre afin d'en mesurer les implications dans l'enseignement moral et civique.

Rappelons rapidement que ce qu'on désigne communément comme une "crise de l'autorité » est inséparable de la modernité. Elle se manifeste par notre difficulté, voire notre incapacité, à définir l'autorité. Nous ne pouvons plus la penser car nous n'en avons plus aucune expérience commune. La modernité, en développant l'esprit critique 
et en remettant en cause la tradition dans tous les domaines, rompt nécessairement avec l'autorité qui est une forme de pouvoir, bien spécifique et sacralisé. Une société à la fois moderne et sécularisée qui ne conçoit plus son rapport au passé comme fondateur et vivifiant ne peut que la méconnaître, mais, avec elle, ce sont les liens entre générations qui risquent d'être brisés, ce qui signifie rien de moins que la " perte des assises du monde ${ }^{15} »$.

13 À ce recul de l'autorité, s'ajoutent de nouvelles modalités de sa régulation sociale, moins «verticales" et plus «horizontales ${ }^{16}$ ». Au début du $\mathrm{xx}^{\mathrm{e}}$ siècle, la société démocratique, industrielle et rationalisée privilégie les régulations verticales: les normes sociales, produites par les élites, se diffusent d'échelon en échelon et sont intériorisées par tous et dans toutes les classes sociales ${ }^{17}$. À partir des années 1970, elles sont contestées au profit de régulations horizontales qui produisent des normes souples, adaptables, locales. Cette opposition est cependant à nuancer. Dans les faits, les deux types de régulation se combinent, la société privilégiant un style plutôt qu'un autre. Cependant, tandis que les sociétés où la régulation verticale l'emporte offrent à leurs membres une grande stabilité, la lisibilité de normes claires et assumées, et permettent aussi l'expression de l'agressivité qui y trouve des cibles, puisque les responsabilités y sont clairement définies, celles qui développent les régulations horizontales valorisent l'informel, la spontanéité, la flexibilité mais ne sont pourtant pas sans effets pervers. Elles favorisent certes un progrès démocratique et développent le dialogue, mais n'abolissent ni les rapports de domination ni les procédures de contrôle. Par ailleurs, les négociations se font à l'intérieur de cadres cognitifs et symboliques qui, invisibles, ne peuvent être discutés. En fait, les individus sont amenés à se contrôler insidieusement les uns les autres, produisant ainsi des normes à leur insu (comme dans les open spaces). Le contrôle y prend ainsi une forme plus indirecte, enveloppante, plus "maternelle", dans un monde où la figure du père tend à s'estomper. En outre, comme les individus sont censés produire eux-mêmes leurs règles, les antagonismes se muent en une "douce violence symbolique", comme le disait Pierre Bourdieu, cachée et niée. Ne pouvant s'en prendre à personne, notamment à leurs "supérieurs", les agents "autonomes " et "responsables" ont tendance à diriger leur agressivité vers leurs pairs ou à retourner la violence contre eux-mêmes, tandis que les anciennes solidarités, comme les collectivités de travail, s'estompent.

14 Ainsi ce modèle "rationnel-négocié ${ }^{18}$ " laisse dans un certain flou les normes qu'il promeut, ce qui génère instabilité, insécurité et perte de repères. Il importe pour notre propos de souligner que le retrait de la parole, au moment même d'ailleurs où certains prétendent bruyamment "restaurer les valeurs ", relève d'un effet de système. Là où, de fait, la contestation ne peut même plus s'exprimer puisqu'il n'y a plus rien à contester, ni aucune autorité obvie à qui s'opposer, c'est moins à l'émergence d'une société démocratique qu'on assiste qu'à celle d'une société gestionnaire, société prétendument sans crise, que je qualifierais de «cybernétique » dans la mesure où elle est censée s'auto-réguler, sans que les normes qui président à cette régulation ne soient réellement explicitées : comme dans les systèmes fermés où la régulation se fait par " feed-back » ou " rétro-contrôle » (comme pour la régulation hormonale des organismes vivants ou pour la température des appareils de chauffage dotés d'un thermostat), la norme régulatrice existe néanmoins, bien qu'une fois activée, elle n'intervienne plus. Elle est pourtant située en dehors du système de régulation et n'est pas fixée par lui. La société «fonctionne » ainsi comme un automate, sans heurts visibles, puisqu'il n'y a plus d'autorités clairement identifiables pour en affirmer les valeurs ${ }^{19}$, pour en assumer 
la responsabilité et contre lesquelles s'insurger (c'est en ce sens qu'on parle aujourd'hui de "gouvernance » et non plus de "gouvernement»), sans qu'on y ait vraiment gagné en liberté.

Ces évolutions à la fois culturelles, sociales, politiques doivent, me semble-t-il, être mises en rapport avec la difficulté où nous sommes de concevoir, de formuler et donc de transmettre une morale, en l'absence de règle générale faisant consensus ${ }^{20}$. Dans une telle ambiance, il ne s'agit plus, pour les individus, d'agir par «pur respect de la loi morale ", c'est-à-dire de manière véritablement autonome (la loi a été intériorisée et les individus se la proposent à eux-mêmes), ni même "conformément » à la loi et dans "l'hétéronomie ", mais seulement de "fonctionner" de manière optimale. L'idée même de morale ne fait plus sens et c'est pourquoi nous sommes confrontés, à un " rejet de principe d'une démarche morale et, a fortiori, d'une éducation morale ${ }^{21}$ ", puisque tout le dispositif culturel et social qui sous-tendait une certaine évidence en matière de morale a été bouleversé. D'une part, chacun étant à l'origine de ses propres normes, qui ne vaudraient que pour soi et que nul ne prétend imposer à personne, aucun magistère moral ne peut être tenu, pas même par les parents, les éducateurs et encore moins par les professeurs. La morale a perdu le caractère prescriptif qui la constituait. Elle ne saurait donc être ni enseignée, ni instituée. D'autre part, puisqu'il n'y a plus de transmission possible, la notion même de "conscience morale ", comme instance d'intériorisation de la loi devient incompréhensible et, avec elle, cet « idéal du gouvernement de soi », supplanté par un nouvel «idéal d'authenticité ${ }^{22}$. Désormais, il n'y a de place que pour une éthique minimale qui serait, au mieux, le fruit d'une négociation à l'intérieur d'un groupe.

Ce mythe d'une société autorégulée va donc de pair avec celui de l'individu autoproduit et auto-fondé. En effet, cette crise de l'autorité et de la transmission est particulièrement sensible dans le domaine de l'éducation et de l'enseignement. À l'École, l'apprentissage semble avoir définitivement supplanté la transmission ${ }^{23}$. Le modèle dominant aujourd'hui est au contraire celui de l'apprenant acteur de son apprentissage et constructeur de son propre savoir. Nous ne développerons pas les objections épistémologiques que l'on peut lui adresser ${ }^{24}$. Remarquons cependant que s'il exerce sur nous une telle séduction théorique et que s'il a pu s'imposer assez rapidement et avec une telle force, c'est qu'il correspond sans doute aux aspirations profondes de notre société, mais peut-être aussi à ses mythes. Notons, par ailleurs, qu'il existe toujours des transmissions résiduelles qui, bien que désavouées, continuent à agir, même si c'est dans un rapport de maître à disciple. Néanmoins, toutes ces évolutions apparaissent comme des épiphénomènes face à ce qu'il est convenu d'appeler la "révolution numérique ", tant elle semble balayer tout sur son passage, de l'idée même d'école à la nécessité d'apprendre. En effet, si tout le savoir est "à portée d'un clic » comme le disent les messages publicitaires, il n'y a plus, apparemment, d'instruction ou de médiation nécessaires et on pourrait dès lors prédire, et pour certains souhaiter, la «fin de l'école ${ }^{25}$ ». De nouveau, c'est la transmission du savoir verticale et académique qui est mise à mal au profit d'usage plus horizontaux, collaboratifs et amateurs, mais où tous les niveaux et les types de messages sont mis sur le même plan, sans hiérarchie, avec donc un risque de confusion et d'anarchie. En fait, le numérique révèle, catalyse et accélère des processus qui travaillent en profondeur et lentement la société et qui bousculent la fonction de professeur et les missions de l'école. Or, tout n'est pas «numérisable». Dans l'un comme dans l'autre cas, ces problématiques nouvelles font saillir la nécessité d'une transmission délibérée et 
permettent de mieux en saisir les enjeux et la nature. Certes, les techniques, les pratiques pédagogiques « innovantes ", la « culture numérique ", profondément hostile à l'idée même d'autorité, chamboulent la posture du maître et semblent le destituer : de professeur, il devient animateur, modérateur ou tuteur ${ }^{26}$.

\section{D'un « partage transitionnel » de la littérature dans l'espace transférentiel de la classe}

17 Pourtant il y a bien un "effet-maître ». Malgré tout, il y a encore de la transmission. Dans les classes où le cours magistral a disparu depuis bien longtemps et où il ne saurait être question de le rétablir, des élèves, des étudiants se réjouissent encore qu'on leur parle et ils apprennent mieux ainsi parce qu'il y a alors un rapport au savoir médiatisé par un professeur, parce que la connaissance s'incarne dans une personne. Il $\mathrm{y}$ a là un sujet dont la parole est porteuse non seulement d'informations mais de savoir, c'est-à-dire de connaissances qui ont un sens susceptible d'orienter une existence. Ce sujet s'adresse à d'autres sujets, avec lesquels il peut nouer des relations dans lesquelles la dimension affective et l'identification ne sont pas absentes, parce qu'il y a du transfert, des projections imaginaires et une inscription dans du symbolique. De même, alors que l'école semble devenue obsolète, les pouvoirs publics, les parents, les éducateurs et les enfants eux-mêmes plébiscitent sa dimension éducative et sociale. C'est cette circulation de la parole qui se retrouve dans l'espace de la salle de classe ou de l'amphithéâtre universitaire. Nous pouvons suivre ici les analyses très suggestives d'Yves Citton ${ }^{27}$. Ce qui s'y joue peut être compris comme une cérémonie ou une célébration collective. En effet, alors que nous pouvons jouir de nombreux événements culturels, confortablement installés chez nous, nous continuons à fréquenter les théâtres, les salles de concert ou les stades. Nous y cherchons le partage d'une émotion. Être ensemble, de façon réelle et pas seulement virtuelle, devant un spectacle vivant, nous permet de ressentir vraiment parce que nous prenons alors conscience de ce que la performance de l'artiste ou du sportif a d'unique, de non reproductible. De plus, le comédien ou le musicien ne peuvent faire cette prestation-là que parce qu'ils sont portés par les autres comédiens et les autres musiciens et aussi par le public à ce moment-là. De même, un véritable cours comprend toujours une part d'échanges et d'improvisation, ce qui fait la valeur d'un véritable professeur, d'un bon professeur, qui est toujours l'auteur de son cours mais qui, comme le dramaturge, ne peut tenir sa partie sans un public: en ce sens, tous les enseignants sont des "enseignantschercheurs » qui s'instruisent avec leurs élèves ${ }^{28}$. Ainsi l'espace de la classe apparaît aussi comme un espace transitionnel où du transfert s'opère, ce qui rend possible une transmission.

Ceci est patent quand le professeur lit et commente un texte littéraire avec ses élèves. Nous entendrons ici « littérature » et « textes littéraires » en un sens très large, allant de la bande dessinée, des albums pour les plus petits et de la littérature jeunesse, aux mythes, en passant par les contes et les fables. En effet, le principal intérêt de l'étude de la littérature ${ }^{29}$, en ce sens, c'est qu'il ne peut y avoir d'étude de texte sans commentaire de texte et donc sans interprétation ou, pour mieux le dire, que lire c'est toujours et d'emblée interpréter (de même qu'écrire). Autrement dit, l'étude d'un texte littéraire permet d'accueillir la pluralité des lectures et des sensibilités, mais crée en même temps du commun à l'intérieur même de cette diversité. Or, le principal danger qui 
guette un enseignement moral et civique est le risque d'imposer, y compris à son insu, une vision du monde, et c'est, on l'a vu, cette crainte légitime qui retient bien souvent les éducateurs, sans parler des enseignants. Il faut donc se garder de deux écueils: l'abstention prudente d'une part, l'imposition plus ou moins dogmatique de l'autre, d'un côté le silence qui abandonne, de l'autre la prescription qui enferme, ici excès de scrupule qui délaisse, là absence de scrupule qui aliène. La littérature ouvre une voie médiane. Parce qu'elle suppose l'interprétation, la lecture d'un texte littéraire fait circuler la parole et permet de combiner la verticalité de la parole du maître qui a choisi le texte, qui le lit à voix haute, qui l'explique et l'horizontalité des échanges avec les plus jeunes et entre les plus jeunes. Mais cette présentation est encore défectueuse. En réalité, le professeur commente le texte avec ses élèves; il n'est pas « celui qui parle devant » eux, mais avec eux, il n'est jamais «celui qui enseigne en public », mais celui qui enseigne à son public, avec lui. Sa parole l'engage et c'est une parole qui s'adresse à d'autres, à l'autre : c'est donc véritablement une parole. Il faut aller encore plus loin, puisque «faire cours» ce n'est pas "faire un cours ${ }^{30}$ ». Sans doute, l'explication de texte a été minutieusement préparée à l'avance, mais elle se métamorphosera au fur et mesure qu'on la produira et c'est alors qu'elle se fera. Tous les enseignants en ont fait l'expérience. Il y aura des impasses, des chemins de traverses, des raccourcis, l'ouverture de nouvelles voies, un cheminement différent d'heure en heure, de classe en classe et c'est pourquoi de nombreuses critiques de l'enseignement dit « transmissif » ou « frontal » manquent souvent l'essentiel. Ce qui se passe est à chaque fois unique. Dans cet exercice, les élèves font donc l'expérience d'un temps dilaté : la lecture partagée rassemble des significations passées, mais dévoile aussi des sens nouveaux, peut-être inédits. Autrement dit, cet acte ne met pas seulement en relation un adulte et des jeunes, il lie aussi les générations en explorant différentes couches interprétatives et en inventant d'autres approches que d'autres suivront, peut-être, un jour... C'est ce qu'on appelle la culture. Et c'est en passant par elle, en en faisant l'expérience, que des valeurs peuvent être transmises, sans être imposées, ce que Guy Coq formule ainsi :

On n'enseigne pas directement les valeurs. Elles présentent un accès souvent indirect, par des médiations culturelles et historiques. Le désir de transmettre les valeurs les hypothèque chaque fois qu'il n'est que le masque d'une volonté d'empire sur autrui pour le modeler ${ }^{31}$.

19 Cependant, cet usage de la littérature dans l'enseignement moral et civique n'est pas une tactique de contournement. S'il permet de lever de nombreux obstacles, c'est bien en s'appuyant sur la littérarité elle-même, ce qui fait qu'il y a littérature, et sur ce qui se passe dans la lecture. La littérature est plus qu'un "support pédagogique » ou une « ressource». Il ne s'agit pas de prendre les textes comme prétextes et de s'en servir comme tremplin pour ensuite réfléchir et passer, en quelque sorte, aux questions " sérieuses ». On tiendrait alors la littérature en bien piètre estime et on voit mal quel profit on pourrait tirer d'une étude littéraire appauvrie à laquelle ferait suite, on peut le redouter, un bavardage tenant lieu de philosophie. Tout d'abord, une analyse attentive des textes est nécessaire. Pédagogiquement, il faut bien s'assurer de la compréhension littérale du texte, avant de tenter interprétation puis spéculation. Tâches inséparables, en vérité, puisque lire un texte, le comprendre, c'est être capable de l'interpréter, ce qui lui donne un sens. Ainsi, fondamentalement, c'est dans la matière même d'un texte littéraire, qui conduit l'acte de lecture, qu'on peut trouver la substance d'une culture morale. Examinons-en quelques aspects. 
Il faut revenir d'abord à la scène primitive de la littérature. "Raconte-moi une histoire ", demande le petit enfant. On lui raconte une histoire. Il ne sait pas encore lire, à peine parler. Premier contact avec la littérature. Retour aux sources de l'humanité, aussi : premiers récits, mythes, histoires initiatiques, textes qui donnent un sens à l'existence, expliquent le monde, indiquent comment y vivre. Sans y prendre garde, on fait ainsi entrer l'enfant dans un monde de significations, de symboles. On lui donne des modèles. Il s'identifie à des personnages, s'interroge sur les actions des protagonistes, se demande ce qu'il ferait dans telle ou telle situation. Son héros connaît la peur, mais la surmonte : l'enfant y retrouve ses angoisses familières, mais aussi les moyens de les vaincre. Pas de morale abstraite ici, mais des valeurs incarnées, dynamisées et dramatisées par le récit. Les dilemmes moraux y sont explicités: Yabouba doit-il tuer «sans gloire » le lion blessé et " passe[r] pour un homme aux yeux de [s]es frères » ou lui « laiss[er] la vie sauve et, à [s]es propres yeux, [sortir] grandi, mais banni »? On comprend dès lors pourquoi les élèves deviennent si subitement attentifs quand on leur raconte une histoire; même les adultes y prennent plaisir. La narration nous sécurise instantanément. Nous savons bien que ce ne sont que des histoires, nous ne nous en laissons plus conter depuis longtemps, et pourtant elles nous apaisent. Une histoire, c'est de l'éthique en acte : l'histoire se charge de répondre à nos questions. Il y a une "morale de l'histoire». Ces dimensions sont inséparables de l'interprétation qui fait intervenir l'imagination. C'est ce que faisait apparaitre Paul Ricœur en montrant que la narration permet de sortir de la situation tragique du jugement par l'intervention de l'imagination, en ouvrant, par l'interprétation, l'espace des possibles :

C'est dans l'imagination que d'abord se forme en moi l'être nouveau. Je dis bien l'imagination et non la volonté. Car le pouvoir de se laisser saisir par de nouvelles possibilités précède le pouvoir de se décider et de choisisir ${ }^{32}$.

21 Cette fonction d'invention morale et politique de la fiction doit être réévaluée ; elle est manifeste dans l'utopie qui, pour Paul Ricœur, a une «fonction libératrice » et qu'il nous invite à prendre en considération en ces termes :

Imaginer du non-lieu, c'est maintenir ouvert le champ du possible. Ou, pour garder la terminologie que nous avons adoptée dans notre méditation sur le sens de l'Histoire, l'utopie est ce qui empêche l'horizon d'attente de fusionner avec le champ de l'expérience. C'est ce qui maintient l'écart entre l'espérance et la tradition $^{33}$.

Ce faisant la littérature nous redonne du pouvoir sur nos vies. En ce sens, la part du récit dans l'historiographie peut être envisagée sous un jour nouveau. Certes, l'historien ne raconte pas des histoires, mais, en même temps, quand il inscrit les événements du passé dans un récit, quand il les met en intrigue, il leur donne un sens, il les interprète aussi. Il suscite dès lors notre intérêt en nous montrant qu'il y a encore des événements, de l'Histoire, de l'action humaine et libre. Paul Ricœur réhabilite ainsi l'histoire narrative et événementielle contre la "nouvelle histoire», histoire de la longue durée, peut-être plus scientifique, mais peut-être aussi désespérante :

Un temps long peut-être un temps sans présent, donc aussi un temps sans passé ni futur: mais alors ce n'est plus un temps historique, et la longue durée reconduit seulement le temps humain au temps de la nature [...]. C'est que la découverte de la longue durée peut exprimer l'oubli du temps humain, qui requiert toujours le repère du présent. Si l'événement au souffle court fait écran à la prise de conscience du temps que nous ne faisons pas, la longue durée peut aussi faire écran au temps que nous sommes ${ }^{34}$. 
Il en est de même de l'imagination métaphorique, autre élément constitutif d'un texte littéraire, qui stimule elle aussi l'interprétation et relance l'invention et l'action, tout d'abord parce qu'elle suspend momentanément, avec nos convictions, un rapport au monde englué :

[C'est] un libre jeu avec des possibilités, dans un état de non-engagement à l'égard du monde de la perception ou de l'action. C'est dans cet état de non-engagement que nous essayons des idées nouvelles, des manières nouvelles d'être au monde ${ }^{35}$.

24 Les textes allégoriques ou les fables se prêtent particulièrement à cet usage. Les figures y condensent de nombreuses significations mais les déplacent aussi. La lecture se promène alors de signifiants en signifiants ou de signifiants en signifiés. Chacun peut y projeter son intériorité ou ses expériences. Le professeur trouvera donc dans cette riche substance, spécifiquement littéraire, matière à débloquer la parole, à libérer un questionnement, à interroger des valeurs sans se perdre dans l'abstraction, ni pontifier ou se poser en tuteur ou en directeur de conscience.

J'ai ainsi pu assister, au cours d'une visite en $\mathrm{CM} 2$, à une très belle séance autour de la fable de La Fontaine « Le chien et le loup », dans le cadre d'une séquence sur les valeurs républicaines. Après une Discussion à Visée Philosophique laborieuse autour du sujet «Être libre, est-ce faire ce qu'il nous plaît?", où les élèves n'étaient pas parvenus à problématiser la question et à en percevoir les enjeux, les enfants ont pu s'emparer de la notion de liberté, qui dès lors fait sens pour eux, à partir du moment où ils ont pu projeter différentes interprétations, mais aussi des émotions vécues, sur les deux animaux, en particulier la peur des chiens de vagabonds, chiens-loups. De proche en proche, en bondissant d'évocations en évocations, ils en sont venus à interroger les liens entre liberté et marginalité, responsabilité et civisme, société et solidarité, pour finir par articuler liberté, égalité et fraternité, ce qui était bien le but du cours, obtenu ainsi sans faire la leçon.

En ce sens, l'étude des textes littéraires institue ce que Hélène Merlin-Kajman appelle un " partage transitionnel ${ }^{36}$ " où les individualités peuvent s'exprimer sans s'aliéner, mais sans non plus être enfermées dans leur particularité et dans leur solitude. Elles découvrent qu'on peut faire corps sans exclure, qu'on peut s'entendre et être écouté sans avoir besoin de crier. Le texte, comme support de projections, permet à chacun, au sein de la communauté des lecteurs, de dévoiler un peu de son monde intime sans exhibition et découvrir celui de l'autre sans intrusion. Comme le doudou pour le toutpetit, il permet d'aller de l'un à l'autre, de dépasser son angoisse, d'affronter le monde, de surmonter ses traumatismes. Il y a bien pourtant ici des enjeux moraux et politiques évidents. En premier lieu, on peut supposer que les élèves qui auront fait de telles expériences de lecture continueront à lire et à aimer lire et puiseront ainsi, dans la littérature, des ressources pour mieux vivre. Ils seront ainsi moins violents. Ils auront appris à identifier et à exprimer leurs émotions et à reconnaître celles des autres. Or nous savons à quel point la méconnaissance et le mépris pour les affections des victimes, la capacité des bourreaux à se couper de leurs émotions sont des éléments dynamiques dans les processus de destruction. C'est d'ailleurs pour cette raison que le programme d'EMC à l'école élémentaire a mis l'accent sur l'importance d'une éducation affective. Par ailleurs, ces échanges où chacun peut être reconnu et entendu renforcent et développent la subjectivité et, avec elle, une véritable autonomie. C'est encore un enjeu politique essentiel, car, comme Michel Terestchenko le montre à la suite de nombreux travaux d'historiens ou de psychologues ${ }^{37}$, les personnalités constituées, 
fortement étayées, où les différentes dispositions humaines s'harmonisent, sont aussi celles qui sont capables de se lever et de résister, de faire preuve de conduites altruistes, tandis que les êtres fragiles, ceux qui demeurent absents à eux-mêmes, véritables poupées de chiffon, incapables de se poser comme sujets libres, conscients et autonomes, sont aussi ceux qui ont la plus grande propension à se fondre dans la masse, à se soumettre aux ordres sans réflexion, à commettre le pire avec zèle et délectation sadique, car ce n'est que là qu'ils trouvent un minimum de consistance et de jouissance. Alors que les premiers font face au réel, les seconds y sont pris.

\section{En guise de conclusion}

Ces quelques suggestions, nous invitent donc à envisager les études littéraires non comme un supplément d'âme pour mondains oisifs et distingués, mais bien comme un moyen de nous sauver. Ceci nous oblige peut-être à reconsidérer le choix des œuvres que nous faisons étudier aux élèves, à renoncer à un commentaire autoréférentiel, à l'idée que les textes littéraires seraient autotéliques, mais surtout à engager notre parole et à prendre nos responsabilités éducatives. Cependant, il ne s'agit pas de prodiguer un enseignement des lettres au rabais, d'abandonner l'analyse attentive des textes ou de les vider de leur substance littéraire puisque c'est justement par leur littérarité, parce qu'ils sont interprétables, parce qu'ils racontent des choix de vie, parce qu'ils mettent en intrigue et en images des idéaux et des valeurs que les textes éduquent et élèvent à la moralité. Ils nous consolent peut-être, nous bercent parfois, mais nous réveillent aussi.

\section{NOTES}

1. M. Gauchet, «Le refus de l'éthique : comment en sortir? » dans E. Favey et G. Coq (dir.), Pour un enseignement laïque de la morale, Toulouse, éd. Privas, coll. « Le comptoir des idées », 2014, p. 68. 2. M. Gauchet, art. cité.

3. G. Coq, Démocratie, religion, éducation, Paris, Mame, 1993, p. 53.

4. R. Debray, L'enseignement du fait religieux dans l'école laïque. Rapport à M. le Ministre de l'Éducation nationale, février 2002. [En ligne] : < http://media.education.gouv.fr/file/91/4/5914.pdf >

5. J. Ferry, lettre aux instituteurs du 17 novembre 1883.

6. N. Sarkozy, discours du Latran du 20 décembre 2007.

7. H. Arendt, "La crise de l'éducation », dans La Crise de la culture [1968], trad. col., Paris, Gallimard, 1972, p. 244.

8. Par commodité, j'utiliserai l'abréviation EMC pour désigner l'Enseignement moral et civique tel qu'il est mis en œuvre depuis la rentrée 2015 à l'école, tandis que je parlerai d'« enseignement moral et civique » quand je désignerai cette question de manière plus générale.

9. Et qui expliquent les réserves exprimées à ce propos par l'Inspection de philosophie. Voir JeanYves Château, « Des expériences de débat à l'école et au collège : discussion à visée philosophique ou pensée réflexive?», intervention au colloque de Ballaruc des 26, 27 et 28 mars 2003, actes publiés par la DESCO en juin 2004. 
10. Selon la jolie expression d'O. Mongin, voir « Mal d'enfance », dans Esprit, $\mathrm{n}^{\circ} 168$, janvier 1991, p. 62-72. Voir aussi E. Deschavanne et P.-H. Tavoillot, Philosophie des âges de la vie, Grasset, 2007.

11. B. Stiegler, Prendre soin de la jeunesse et des générations, Paris, Flammarion, 2008, p. 14.

12. M. Gauchet et G. Swayn, "Un nouveau regard sur l'histoire de la folie », Esprit, $n^{\circ} 83$, nov. 1983, p. 80.

13. M. Gauchet, La Religion dans la démocratie, Paris, Gallimard, coll. « Folio essais », 2001, p. 105.

14. G. Coq, Démocratie, religion, éducation, Paris, Mame, 1993, p. 82.

15. H. Arendt, "Qu'est-ce que l'autorité » dans La Crise de la culture, ouvr. cité, p. 124. Sur cette question voir aussi Fr.-X. Bellamy, Les Déshérités ou l'urgence de transmettre, Paris, Plon, 2014.

16. J.-D. Reynault, Les Règles du jeu, L'action collective et la régulation sociale, Paris, A. Colin, 1997 et

J. de Munck, «Les métamorphoses de l'autorité », Autrement, n 198, octobre 2000, p. 27.

17. Processus d'intériorisation que Durkheim appelle « l'éducation morale».

18. J. de Munck, art. cité, p. 35.

19. Il convient, bien entendu, de distinguer les «normes » des «valeurs », terme d'ailleurs très ambigu. Je voudrais seulement évoquer ici comment la notion de norme sociale, qui est relative, s'est substituée à celle de valeur morale, qui comporte une exigence d'universalité.

20. Sans, bien sûr, confondre le social, le politique et la morale.

21. M. Gauchet, « Le refus de l'éthique : comment en sortir? », art. cité, p. 68.

22. Ibid., p. 72.

23. Sur ce point, on se reportera notamment à l'ouvrage de M.-Cl. Blais, M. Gauchet et D. Ottavi, Transmettre, apprendre, Paris, Stock, coll. « Pluriel », 2014.

24. Entre autres parce que les savoirs méthodiques ne s'acquièrent peut-être pas de la même manière qu'ils se développent et qu'il n'est pas sûr que la démarche d'élève soit comparable à celle d'un chercheur.

25. F. Durpaire et B. Mabilon-Bonfils, La Fin de l'école, L'ère du savoir-relation, Paris, PUF, 2014.

26. Tout d'abord en ce qui concerne le statut social et économique des enseignants, le développement des MOOC aux États-Unis s'est accompagné d'une érosion vertigineuse des titularisations sur postes universitaires et d'un accroissement sans précédents de docteurs en position d'adjoints et précarisés. Voir Y. Citton, Pour une écologie de l'attention, Paris, Seuil, coll. « La couleur des idées », 2014, p. 142-143.

27. Ibid., p. 149-153.

28. En ce sens, la notion de «transmission » est bien plus riche que celle qui est évoquée à travers des querelles didactiques ou pédagogiques. La «transmission » n'est pas le «transmissif » et elle ne se réduit pas non plus à une imposition, ni même à une inculcation.

29. Je ne me prononcerai donc pas sur ce qui doit être appelé ou non « littérature "; je me réfère à la lecture et à l'étude littéraire, particulièrement dans un contexte scolaire.

30. J.-P. Carlet, «La lecture philosophique en classe entendue comme instruction des élèves. Deux esquisses de cours », L'Enseignement Philosophique, numéro hors-série, «Entrer dans le métier », publié par l'APPEP, 2012.

31. G. Coq, Démocratie, religion, éducation, Paris, Mame, 1993, p. 81.

32. P. Ricœur, Du Texte à l'action, Essais d'herméneutique II, Paris, Seuil, 1986, p. 132.

33. Ibid.p. 391.

34. P. Ricœur, Temps et récit I, Paris, Seuil, coll. «L'ordre philosophique », 1983, p. 395.

35. P. Ricœur, Du texte à l'action, ouvr. cité, p. 220.

36. H. Merlin-Kajman, Lire dans la gueule du loup, Essai sur une zone à défendre, la littérature, Paris, Gallimard, coll. « NRF essais », 2016, p. 18.

37. M. Terestchenko, Un si fragile vernis d'humanité. Banalité du mal, banalité du bien, Paris, La Découverte, 2005. 


\section{RÉSUMÉS}

L'article analyse les causes culturelles d'un retrait de la parole enseignante dans l'enseignement : crise de l'autorité, émergence d'une société gestionnaire et méfiance généralisée envers la transmission, tandis que le numérique catalyse et accélère ce processus. Entre la verticalité de la parole du maître et l'horizontalité des échanges entre élèves, l'explication des textes littéraires est envisagée comme une voie médiane, pour redonner un sens plein à l'engagement de la parole enseignante, dans un espace véritablement transférentiel. Mais cela n'est possible qu'en prenant en compte la littérarité et en donnant un espace didactique à ce qui se passe dans la lecture des élèves. L'article réhabilite l'imagination, définie comme pouvoir de se laisser saisir par les possibles, et rejette l'instrumentalisation des textes comme prétexte, dans l'enseignement moral et civique.

The article analyses the cultural causes of a withdrawal of the teaching voice in education: the crisis of authority, the emergence of a management society and widespread mistrust of transmission, while digital technology catalyses and accelerates this process. Between the verticality of the teacher's speech and the horizontality of exchanges between students, the explanation of literary texts is envisaged as a middle way, to give full meaning to the engagement of the teaching speech, in a truly transferential space. But this is only possible by taking into account literality and by giving a didactic space to what happens in students' reading. The article rehabilitates the imagination, defined as the power to let oneself be seized by the possible, and rejects the instrumentalization of texts as a pretext in moral and civic education.

\section{AUTEUR}

\section{MYRIAM BELMONTE-ESTRADE}

Myriam Belmonte-Estrade est professeure agrégée de philosophie à l'ESPÉ de Grenoble (Université Grenoble-Alpes). Elle a enseigné une vingtaine d'années en lycée et travaille sur la parole enseignante. Elle a publié récemment « La philosophie comme art brut » (Diotime, $\mathrm{n}^{\circ} 79$, 2019) et « Situation de la parole » (Feuillets psychanalytiques $\left.n^{\circ} 2,2017\right)$. 